\title{
Uveal Melanoma Treated with lodine-125 Episcleral Plaque: An Analysis of Dose on Disease Control and Visual Outcomes
}

\author{
Bradford A. Perez, M.D. ${ }^{1,{ }^{\star}}$, Pradeep Mettu, M.D. ${ }^{2,}{ }^{\star}$, Lejla Vajzovic, M.D. ${ }^{2}$, Douglas Rivera, \\ M.D. ${ }^{3}$, Ali Alkaissi, M.S. ${ }^{1}$, Beverly A. Steffey, M.S. ${ }^{1}$, Jing Cai, Ph.D. ${ }^{1}$, Sandra Stinnett, \\ Dr.PH ${ }^{4}$, Jonathan J. Dutton, M.D., Ph.D. ${ }^{5}$, Edward G. Buckley, M.D. ${ }^{2}$, Edward Halperin, M.D., \\ M.A. ${ }^{6}$, Lawrence B. Marks, M.D. ${ }^{7}$, Prithvi Mruthyunjaya, M.D. ${ }^{1,2,{ }^{* \star}}$, and David G. Kirsch, \\ M.D., Ph.D. ${ }^{1,8,{ }^{* *}}$ \\ ${ }^{1}$ Duke University, Department of Radiation Oncology, Durham, NC \\ 2Duke University, Department of Ophthalmology, Durham, NC \\ ${ }^{3}$ Austin Cancer Centers, Austin, TX \\ ${ }^{4}$ Duke University, Department of Biostatistics and Informatics, Durham, NC \\ ${ }^{5}$ University of North Carolina, Department of Ophthalmology, Chapel Hill, NC \\ ${ }^{6}$ New York Medical College, Department of Radiation Oncology, Valhalla, NY \\ ${ }^{7}$ University of North Carolina, Department of Radiation Oncology, Chapel Hill, NC \\ ${ }^{8}$ Duke University, Department of Pharmacology and Cancer Biology, Durham, NC
}

\begin{abstract}
PURPOSE-In the treatment of uveal melanomas, the optimal prescribed dose to maximize disease control, but minimize radiation-related complications is unknown. Historically our institution has treated uveal melanomas to doses less than $85 \mathrm{~Gy}$ to the tumor apex even if the apex was less than $5 \mathrm{~mm}$ in height. Here, we investigate how tumor control and visual outcomes are affected by the radiation dose at the tumor apex.
\end{abstract}

METHODS AND MATERIALS-A retrospective review was performed to evaluate patients treated for uveal melanoma with Iodine-125 plaques between 1988 and 2010. Radiation dose is reported as dose to tumor apex and dose to $5 \mathrm{~mm}$. Primary end points included time to local failure, distant failure, and death. Secondary end points included eye preservation, visual acuity,

\footnotetext{
(C) 2014 Elsevier Inc. All rights reserved

Corresponding Author: David Kirsch, MD, PhD, Associate Professor, Department of Radiation Oncology, Duke University Medical Center, Box 91006 LSRC B233, Durham, NC 27710, Phone: 919-681-8586, Fax: 919-668-1867, david.kirsch@ duke.edu. BAP and PM contributed equally to this work.

** PM and DGK are co-senior authors for this work.

Publisher's Disclaimer: This is a PDF file of an unedited manuscript that has been accepted for publication. As a service to our customers we are providing this early version of the manuscript. The manuscript will undergo copyediting, typesetting, and review of the resulting proof before it is published in its final citable form. Please note that during the production process errors may be discovered which could affect the content, and all legal disclaimers that apply to the journal pertain.

Conflict of Interest Statement: Other conflicts of interest: none
} 
and radiation-related complications. Univariate and multivariate analyses were performed to determine association between radiation dose and the end point variables.

RESULTS-One hundred ninety patients with sufficient data to evaluate the end points were included. The 5 year local control (LC) rate was $91 \%$. The 5 year distant metastases (DM) rate was $10 \%$. The 5 year overall survival (OS) rate was $84 \%$. There were no differences in outcome (LC, DM, OS) when dose was stratified by apex dose quartile (<69 Gy, 69-81 Gy, 81-89 Gy, >89 Gy). However, increasing apex dose and dose to $5 \mathrm{~mm}$ depth were correlated with greater visual acuity loss ( $\mathrm{p}=0.02, \mathrm{p}=0.0006)$, worse final visual acuity $(\mathrm{p}=0.02, \mathrm{p}<0.0001)$ and radiation complications $(\mathrm{p}<0.0001, \mathrm{p}=0.0009)$. In addition, enucleation rates were worse with increasing quartiles of dose to $5 \mathrm{~mm}(\mathrm{p}=0.0001)$.

CONCLUSIONS-Doses at least as low as $69 \mathrm{~Gy}$ prescribed to the tumor apex achieve rates of local control, distant metastasis free survival, and overall survival that are similar to radiation doses of 85 Gy to the tumor apex, but with improved visual outcomes.

\section{Keywords}

uveal melanoma; choroidal melanoma; Iodine-125 brachytherapy; radiation toxicity; enucleation; dose reduction

\section{INTRODUCTION}

Uveal melanoma is the most common primary malignant ocular tumor in adults with an annual age adjusted incidence of 5.1 per million[1]. In an effort to preserve the globe and thus vision, brachytherapy and particle beam radiotherapy have been used actively as alternatives to enucleation. Stallard popularized episcleral brachytherapy in the 1960's by utilizing the highenergy isotope cobalt-60[2]. Since that time, low energy photon emitting isotopes such as iodine-125 $\left({ }^{125} \mathrm{I}\right)$ and palladium-103 as well as ruthenium-106, a beta emitter, have replaced cobalt- 60 because these isotopes can be easily shielded thereby reducing dose to adjacent ocular adnexal structures and personnel.

The Collaborative Ocular Melanoma Study (COMS) Medium-sized melanoma trial was a North American, multi-institutional, prospective randomized study involving over 1300 patients between 1987-1998, which found that overall survival was similar in patients that received enucleation compared to those patients that underwent globe preserving therapy with ${ }^{125}$ I episcleral plaques[3]. Patients treated with brachytherapy in the COMS protocol received a minimum tumor dose of 85 Gy delivered $5 \mathrm{~mm}$ deep to the inner sclera, or to the maximum apex tumor height, whichever was greater. The prescription dose of 85 Gy was chosen based on expert opinion and experience with plaque brachytherapy for uveal melanoma. Plaque brachytherapy was effective in sterilizing the gross tumor, with local control being achieved in about $90 \%$ of patients. However, radiation-induced ocular injury was not uncommon and necessitated enucleation in about $5 \%$ of patients. Further, among patients retaining their eye, at 3 years post-brachytherapy, $49 \%$ of patients had lost six or more lines of visual acuity from baseline, presumably from late radiation toxicity to the retina and optic nerve[4]. Given the high rate of local control, but relatively high rate of 
toxicity, it is reasonable to consider if a lower radiation dose might yield a more favorable therapeutic ratio.

Few reports have directly compared different radiation doses for ocular melanoma. A randomized study of proton beam radiotherapy evaluating $50 \mathrm{vs.} 70$ cobalt gray equivalents (CGE), each delivered in 5 fractions over 7 days, showed a lower rate of visual field loss in the lower-dose group, but no difference in loss of visual acuity[5]. Tumor control was similar in both arms with only $2-3 \%$ of patients experiencing local recurrence and about $7-$ $8 \%$ of patients with distant metastases at 5 years after irradiation[5]. Utilizing ${ }^{125}$ I plaque brachytherapy, retrospective analyses suggest that prescribed doses lower than in the COMS group trial can achieve similar rates of disease control with lower rates of radiation toxicity[6,7]. At our institution, we have prescribed doses ranging from $63-85$ Gy to the tumor apex even when tumors were $<5 \mathrm{~mm}$ in height. A lower prescription dose prescribed to a shorter height results in a lower total radiation dose to the tumor apex as well as to the normal structures in the eye (Supplemental Figure e1). Here, we report outcomes for this treatment approach in 190 patients with ${ }^{125}$ I plaque brachytherapy.

\section{METHODS AND MATERIALS}

\section{Treatment Method}

As part of an Institutional Review Board-approved study, the records of patients treated at Duke University Medical Center for primary uveal melanoma of the choroid and/or ciliary body with ${ }^{125}$ I eye plaque brachytherapy between $1988-2010$ were retrospectively reviewed. Patients were identified for review and potential inclusion in the study through the Duke Radiation Oncology Database and the Duke Tumor Registry. Patients were excluded from review if they had tumors involving the iris (as in the COMS study[8]), had metastatic or recurrent disease at presentation, or had incomplete data to review for inclusion in the study. Patients with less than 1 year of follow up were excluded unless they met one of the primary endpoints within the first year.

All patients were initially evaluated and diagnosed with uveal melanoma by an ophthalmologist with expertise in ocular oncology (J.J.D, E.G.B., and P.M.). Each study patient underwent a comprehensive baseline ophthalmic evaluation with measurement of visual acuity (Snellen or ETDRS acuity), complete anterior and posterior segment examination and ancillary testing, when appropriate, that included fundus photography, fluorescein angiography, and A and B-scan ultrasonography. Tumor height and diameter were determined clinically and confirmed with fundus photographs and standardized echography. A baseline systemic evaluation to exclude metastatic disease included liver function tests, chest X-ray or chest CT, and focal imaging of the abdomen with either abdominal/pelvic CT or ultrasound.

Our institution was a COMS group trial participant and under that guidance, episcleral plaques were obtained and utilized per the COMS group specifications that have been previously published[8]. Notched, ciliary, and circular rimmed plaques with diameters of 12 , $14,16,18$, and $20 \mathrm{~mm}$ were used with Silastic seed carriers carrying $8,13,13,21$, and $24{ }^{125}$ I seeds in a predesigned pattern, respectively. ${ }^{125}$ I seeds (Model 6711) were obtained 
on an individual basis from Oncura manufactured by Medi-Physics, Inc. (Arlington Heights, IL). Plaques were chosen to provide a $2 \mathrm{~mm}$ margin around the tumor edge (i.e. $4 \mathrm{~mm}$ larger than the maximal tumor diameter), but a $20 \mathrm{~mm}$ plaque was used for 4 patients with a tumor diameter greater than $16 \mathrm{~mm}$. Preplanning and seed activity verification was performed prior to each plaque placement. Intraoperative tumor localization (via direct visualization or transillumination) was followed by dummy plaque placement to ensure adequate tumor coverage prior to suturing of the radioactive plaque. Following the duration of prescribed plaque dwell time, typically approximately 120 hours, plaques were removed again in the operating room.

Our series included patients that were, and were not, on the COMS study and our prescribed doses, and dose prescription points, varied over time. For patients enrolled on the COMS protocol, the COMS prescription recommendations were followed. Prior to participating in the COMS trial, doses less than 80 Gy were generally prescribed to the tumor apex irrespective of the tumor height. Following the COMS trial publication, doses between 8085 Gy were utilized although the prescription height was maintained at the tumor apex height. Similarly, patients on the COMS study had follow-up evaluations as dictated by the study. Those patients not on study had similar follow up evaluations. Typically, patients returned for follow-up with the ophthalmologists within 1-week post removal of the plaque, then generally every 3 months for the first year, every 6 months for 2 years, and then yearly thereafter. Examinations included a full ophthalmologic exam with visual acuity testing, retinal examination, and ancillary testing including standardized echography. In addition, liver function testing and surveillance imaging was performed yearly or as clinically indicated.

\section{Dose Calculations and Conversions}

For the purposes of this study, when the prescription height was different than the apex height, the dose at the tumor apex was calculated and reported here. We initially followed the original dose calculation formalisms adopted by COMS in 1986. In 1996 COMS adopted the recommendations of the Radiation Therapy Committee of the American Association of Physicists in Medicine (AAPM) Task Group \# 43(TG-43)[9] in anticipation of a revised calibration standard for ${ }^{125}$ I seeds. Dosimetry calculations with the newer data resulted in a significant change to the stated dose. Recalculations suggested that the original calculations were overestimated by as much as $15 \%$ [10]. These recalculations resulted in a reported reduced absorbed dose at the prescription point. We did not adopt the TG-43 formalism until November of 1997. Therefore, dose data from patients treated prior to this, were recalculated using the TG-43 recommendations[9]. Dose and dose rates reported here utilize the TG-43 assumptions therefore lending themselves to direct comparison of the COMS group data. Supplemental Table e1 outlines how dose was recalculated to assure similar comparisons across different prescribing practices and dose calculation eras. For tumor control endpoints, we compare dose delivered to the tumor apex. For toxicity endpoints, we describe dose delivered to the tumor apex, sclera, and dose to $5 \mathrm{~mm}$ depth beyond the sclera. We selected dose to $5 \mathrm{~mm}$ to serve as a normalization point for comparing dose to normal tissues between patients because it was the minimum prescription depth for patients in the COMS study. 


\section{Endpoints and Statistical Analysis}

Visual acuity was collected at baseline and at the date of last follow-up. Data related to the endpoint variables were collected by retrospective evaluation of the clinical charts or through the Duke Tumor Registry. Other data extracted from the chart included patient age, basal tumor dimensions and apex tumor height, tumor location by quadrant and anterior/ posterior location, prescription height and dose, and plaque size, shape and seed strength to recalculate apex dose.

Primary end points included time from plaque placement until local failure, distant failure, and death. Local failure was defined as secondary enucleation due to tumor growth, regrowth after an initial period of tumor regression requiring adjuvant laser therapy, or a lesion without any size regression after treatment when the follow-up period was at least 6 months. Distant failure was defined as the first occurrence of metastatic disease diagnosed by surveillance imaging or clinical examination. Combined failure was defined as both local and distant failure and such patients were analyzed in both the local failure and distant failure analyses. Secondary end points included time to eye preservation, visual acuity, and radiation complications including retinopathy, optic neuropathy, cataract, neovascular glaucoma, and keratopathy. Change in visual acuity was measured as lines of visual acuity lost.

Life table analysis with the log-rank test[11] was used to identify characteristics that were associated with the outcomes. For the purposes of this study, apex dose was divided into quartiles ( $\left.1^{\text {st }}-<69 \mathrm{~Gy} ; 2^{\text {nd }}-69-81 \mathrm{~Gy} ; 3^{\text {rd }}-81-89 \mathrm{~Gy}, 4^{\text {th }}->89 \mathrm{~Gy}\right)$ to evaluate the effect of varying dose on study endpoints. Dose to $5 \mathrm{~mm}$ depth was also divided into quartiles ( $1^{\text {st }}$. $<53 \mathrm{~Gy} ; 2^{\text {nd }}-53-66 \mathrm{~Gy} ; 3^{\text {rd }}-66-85 \mathrm{~Gy}, 4^{\text {th }}->85 \mathrm{~Gy}$ ). Univariate and multivariate analyses on characteristics associated with the end point variables were performed using the Cox proportional hazards model[12]. Variables that were identified as significant on univariate analyses were entered in the multivariate analyses. Visual acuity statistics utilized analyses of variance, contingency tables, and two sided student's t-tests as appropriate to evaluate significance.

\section{RESULTS}

\section{Patient, tumor, and dose characteristics}

One hundred ninety patients with primary unilateral uveal melanoma were included. Patient and tumor characteristics are described in Table 1. Most of the tumors included in the study were Medium sized according to the COMS staging system [13]. The median tumor apex height was $3.9 \mathrm{~mm}$ (range $1.5-10.8 \mathrm{~mm}$, interquartile range 3-5.4 $\mathrm{mm}$ ) and the median maximal tumor diameter was $11.0 \mathrm{~mm}$ (range $4.7-17.5 \mathrm{~mm}$, interquartile range $9.5-13 \mathrm{~mm}$ ). There was no difference in tumor height $(\mathrm{p}=0.20)$ or maximum basal tumor diameter $(\mathrm{p}=0.09)$ by apex dose quartile. The majority of tumors were located in the posterior pole (71\%) and temporal hemisphere (66\%). The median total dose delivered to the apex of the tumor (as computed for this current analysis) was $81.0 \mathrm{~Gy}$ (range 51.0 - 180.1 Gy, interquartile range 68.7-89.0 Gy). The median duration in years (range; interquartile range) of follow-up was $4.1(0.1-19.3 ; 2.4-5.9)$ for the local failure analysis, $4.3(0.3-19.3 ; 3.0-$ 
6.2) for the distant failure analysis, and $5.9(0.8-22.3 ; 4.2-10.7)$ for the overall survival analysis.

\section{Local Failure}

Local failure was noted in $17(9 \%)$ patients. Four (2\%) patients experienced both local and distant failures. Two of these patients with combined failure were diagnosed concurrently with local and distant failure and two patients developed distant failure subsequent to local failure. The five-year actuarial freedom from local failure rate was $91 \%$ (Figure 1A). Among the 17 patients with local failure, $7(41 \%)$ were managed with transpupillary thermal therapy (TTT), 6 (35\%) underwent enucleation, $1(6 \%)$ underwent treatment with proton radiotherapy, $1(6 \%)$ received TTT followed by proton beam radiotherapy, $1(6 \%)$ was referred to an ocular oncologist at another institution, and $1(6 \%)$ received no local treatment due to concurrent active brain metastases at the time of presentation. Among the 6 patients undergoing enucleation for local failure, the presence of tumor was confirmed by pathology in all patients. There was no difference in time to local failure by apex dose quartile ( $\mathrm{p}=0.26$, Figure 1A). The crude local failure rates by apex dose quartile were $6 \%, 11 \%, 15 \%$ and $4 \%$. Among tumors with height $\geq 5 \mathrm{~mm}(\mathrm{n}=60)$, there was no difference in rate of local failure by apex dose quartile (Supplemental Figure e2A). On univariate analysis, no tested variables were associated with local failure (Table 2).

\section{Distant Failure}

Twenty three (12\%) patients were noted to have a distant failure. The five-year actuarial freedom from distant failure was $90 \%$ (Figure 1B). Among the 23 patients with distant failure, 19 patients were deceased at the time of data collection with a median survival after distant failure of about 4.9 months (range 0-42 months). Four patients were still living with a median follow up after distant failure of 30.0 months (range 4-111 months). Only 1 patient had documented survival longer than 5 years after diagnosis of distant metastatic disease. When grouping the treated tumors by apex dose quartile there was no relationship between dose at the tumor apex and the likelihood of distant failure ( $\mathrm{p}=0.78$, Figure 1B). Among tumors with height $\geq 5 \mathrm{~mm}$, there was no difference in metastasis free survival by apex dose quartile (Supplemental Figure e2B). Of 42 patients with tumors located in the superotemporal quadrant, 12 (29\%) experienced distant failure. On univariate analysis larger maximum basal tumor diameter (MBTD) $(\mathrm{p}=0.03)$ and tumor location by quadrant $(\mathrm{p}=0.03)$ were associated with distant failure. On multivariate analysis, greater MBTD and tumor location by quadrant were not significantly associated with greater risk of distant failure $(\mathrm{p}=0.12, \mathrm{p}=0.07$, Table 2).

\section{Overall Survival}

The 5 year overall survival rate was $84 \%$ (Figure 1C). There was no significant relationship between tumor apex dose and likelihood of overall survival when the tumors were distributed and evaluated by quartile, though there was a trend towards the highest quartile ( $>89$ Gy) being less likely to survive over time ( $\mathrm{p}=0.13$, Figure 1C). Similarly, larger tumors ( $\geq 5 \mathrm{~mm}$ height) treated in the highest apex dose quartile appeared to have worse survival outcomes ( $\mathrm{p}=0.04$, Supplemental Figure e2C). On univariate analysis, older age at procedure $(\mathrm{p}<0.01)$, larger maximum basal tumor diameter $(\mathrm{p}<0.01)$, and higher apex dose $(\mathrm{p}=0.03)$ 
were associated with worse overall survival (Table 2). On multivariate analysis accounting for age at procedure and maximum basal tumor diameter the relationship between decreased overall survival and higher dose to the tumor apex remained significant $(\mathrm{p}=0.02$, Table 2$)$.

\section{Eye Preservation}

Eleven $(6 \%)$ patients underwent enucleation. The five-year actuarial freedom from enucleation rate was 93\% (Figure 2B). Enucleation was performed in 6 of 11 patients due to local failure, in 4 of 11 due to the development of a blind, painful eye, and in 1 of 11 due to media opacity causing inadequate visualization for monitoring in an eye with poor vision. The crude enucleation rates by apex dose quartile were $2 \%, 4 \%, 6 \%$ and $11 \%(\mathrm{p}=0.35$, Figure 2A), respectively. To compare radiation dose to normal tissues across patients, dose to $5 \mathrm{~mm}$ depth beyond the sclera was also evaluated. The crude enucleation rates by $5 \mathrm{~mm}$ dose quartile were $0 \%, 2 \%, 2 \%$ and $19 \%(\mathrm{p}=0.0001$, Figure $2 \mathrm{~B})$. On univariate analysis, higher dose to the tumor apex ( $\mathrm{p}<0.01$, Table 3$)$, to $5 \mathrm{~mm}$ depth $(\mathrm{p}<0.01$, Supplemental Table e2) and to the sclera ( $<<0.01$, Supplemental Table e3) were all associated with time to enucleation. Additionally, greater tumor height was associated with time to enucleation on univariate analysis $(\mathrm{p}=<0.01$, Table 3$)$. Finally, anterior tumor location was associated with increased risk of enucleation ( $p=0.03$, Table 3 ) as 3 of 11 tumors requiring enucleation were located in the ciliary body $(n=15$, Table 1$)$. On multivariate analysis accounting for tumor height and anterior to posterior location, the relationship between enucleation and higher dose to the tumor apex ( $\mathrm{p}<0.01$, Table 3$)$, to $5 \mathrm{~mm}$ depth $(\mathrm{p}<0.01$, Supplemental Table e2), and to the sclera $(\mathrm{p}<0.01$, Supplemental Table e3) remained significant.

\section{Radiation Complications}

The 5 year actuarial risk of documented treatment complication was $73 \%$. The risk of any treatment complication was significantly associated with increasing quartiles of dose to the tumor apex ( $\mathrm{p}<0.0001$, Figure 2C) and dose to $5 \mathrm{~mm}(\mathrm{p}=0.0009$, Figure 2D). Univariate analyses showed that greater tumor height $(\mathrm{p}<0.01$, Table 3$)$ as well as higher apex dose $(\mathrm{p}<0.01$, Table 3), $5 \mathrm{~mm}$ dose $(\mathrm{p}<0.01$, Supplemental Table e $)$, and scleral dose $(\mathrm{p}<0.01$, Supplemental Table e3) were associated with time to radiation complication. Multivariate analyses confirmed associations between time to radiation complication and tumor height $(\mathrm{p}<0.01$, Table 3$)$, higher apex dose $(\mathrm{p}<0.01$, Table 3$), 5 \mathrm{~mm}$ dose $(\mathrm{p}<0.01$, Supplemental Table e2), and scleral dose ( $\mathrm{p}=0.03$, Supplemental Table e3). Times to each individual radiation complication (radiation retinopathy, optic neuropathy, cataract, neovascular glaucoma, and keratopathy) were also evaluated separately by quartiles for apex dose (Supplemental Figure e3) and $5 \mathrm{~mm}$ dose (Supplemental Figure e4). The most common radiation complications were radiation retinopathy and cataract formation.

\section{Visual Outcomes}

Mean pre-operative visual acuity was 20/37 and final visual acuity was 20/160 (p<0.0001). Increasing $5 \mathrm{~mm}$ dose quartiles were associated with greater worsening of visual acuity $(\mathrm{p}=0.0005$, Figure $3 \mathrm{~B})$. Ninety-three $(49 \%)$ patients lost 3 or more lines of visual acuity from baseline to last follow-up. Increasing apex dose quartile was associated with likelihood of 3 or more lines of worsened visual acuity ( $\mathrm{p}=0.04$, Figure $3 \mathrm{C}-\mathrm{left}$ ). Mean apex dose was 84.9 Gy in eyes with 3 or more lines of vision loss versus 79.8 Gy in eyes with less than 3 
lines of vision loss ( $\mathrm{p}=0.02$, Figure $3 \mathrm{C}$-right). Increasing $5 \mathrm{~mm}$ dose quartile was also associated with likelihood of 3 or more lines of vision loss ( $p=0.0003$, Figure 3D-left). A higher mean $5 \mathrm{~mm}$ dose was again observed in patients with loss of 3 or more lines of visual acuity compared to patients with loss of less than 3 lines of visual acuity (83.5 Gy vs. 66.7 $\mathrm{Gy} ; \mathrm{p}=0.0006$, Figure 3Dright). Mean apex dose was 85.9 Gy in eyes with final visual acuity of 20/200 or worse versus 79.9 Gy in eyes with final visual acuity of 20/200 or better $(\mathrm{p}=0.02$, Figure 3E-right). Similarly, when assessing the impact of dose at $5 \mathrm{~mm}$ on visual acuity using the 20/200 cut off, there was a significant association between risk of visual acuity greater than $20 / 200$ and increasing apex dose quartile ( $p=0.0002$, Figure $3 F-l e f t)$. A mean $5 \mathrm{~mm}$ dose of $88.5 \mathrm{~Gy}$ was seen in the group with worse vision compared to $66.1 \mathrm{~Gy}$ in the group with better than 20/200 vision ( $p<0.0001$, Figure 3F-right).

\section{DISCUSSION}

We have performed a retrospective analysis on a series of 190 patients with uveal melanoma treated with episcleral ${ }^{125}$ I brachytherapy with doses ranging from less than 65 Gy to greater than $85 \mathrm{~Gy}$ to the tumor apex. In our study, we found no relationship between dose delivered to the tumor apex and local tumor control or distant metastases. Compared to the COMS trial in which patients received 85 Gy to a minimum of $5 \mathrm{~mm}$ height with 5-year rates of local control and overall survival of $90 \%$ and $81 \%$, respectively [3], in our retrospective series, we observed similar rates of local control and overall survival despite a generally lower prescribed dose of radiation therapy to the tumor apex, which was often less than 5 $\mathrm{mm}$. By stratifying patients into quartiles based on dose delivered to the tumor apex or to 5 $\mathrm{mm}$, we identified a significant relationship between radiation dose and ocular toxicity. The increased risk of enucleation among tumors receiving higher radiation doses and tumors in the ciliary body may be related to radiation-induced scleral melt and ischemia, which is more prevalent among tumors treated with higher scleral dose, with increasing thickness, and with peripheral choroidal or ciliary body location[14]. These results suggest that treating ocular melanomas with a dose to the tumor apex less than $85 \mathrm{~Gy}$ can achieve high rates of local control while better preserving visual function. This may be particularly important for patients with a tumor apex height of less than $5 \mathrm{~mm}$. In the COMS study, all of these patients received $85 \mathrm{~Gy}$ prescribed to $5 \mathrm{~mm}$ height. By prescribing a lower radiation dose to a point less than $5 \mathrm{~mm}$, the dose to $5 \mathrm{~mm}$ height will be substantially less than $85 \mathrm{~Gy}$ (see schematic, Supplemental Figure e1). Our results indicate that this will translate into a lower rate of eye enucleation and radiation retinopathy with improved visual acuity outcome.

Paradoxically, patients treated with the highest dose to the tumor apex had worse overall survival as measured by all-cause mortality. We did not assess disease specific mortality because this outcome was not available for most patients. It is unclear whether the increased mortality observed in patients with the highest radiation dose delivered to the tumor apex is a consequence of an unanticipated systemic effect of treatment or more likely a Type I error of the statistical analysis. Regardless of the explanation, this finding further supports utilizing a lower prescription dose to the apex of uveal melanomas for ${ }^{125} \mathrm{I}$ brachytherapy.

The results from our study are consistent with several other studies where excellent results of tumor control have been reported with radiation doses that are lower than those used in 
the COMS study (Supplemental Table e2) [5-7]. Compared to higher doses of radiation therapy, previous studies have shown that lower doses of radiation for the treatment of uveal melanoma have correlated with lower rates of visual field loss, radiation retinopathy, radiation optic neuropathy, and visually significant cataract formation[5,7]. Our study similarly showed that patients who received radiation doses in the lower quartiles had more favorable enucleation rates, better visual acuity, and fewer radiation complications. This was true whether we analyzed dose delivered to the tumor apex or to $5 \mathrm{~mm}$.

Murray et al retrospectively evaluated 95 patients and noted a lower incidence of radiation complications with a strategy of prescribing to the tumor apex rather than a uniform $5 \mathrm{~mm}$ for tumors with less than $5 \mathrm{~mm}$ of height[7]. Other studies of radiation toxicity with ${ }^{125} \mathrm{I}$ brachytherapy for uveal melanomas indicate that radiation dose to the macula may be associated with poorer post-treatment visual acuity $[15,16]$. Therefore, to minimize the potential loss of visual acuity following episcleral ${ }^{125}$ I brachytherapy, for tumors in close proximity to the macula, it may be important to prescribe a dose of less than 70 Gy to the tumor apex regardless of height. Prescribing this lower dose may be particularly important for patients with poor contralateral eye visual acuity where preserving visual acuity in the treated eye is of critical importance. Our results indicate that such an approach is unlikely to adversely affect tumor control. However, if a lower prescription dose is utilized, then we recommend intraoperative plaque localization with ultrasound should be used to confirm adequate apposition of the plaque to the sclera to prevent sub-optimal dosing to the tumor apex [17].

As with any retrospective study, there are limitations to interpretation of our reported findings. Data collected is only as robust as the documentation available in the clinic charts and the Duke Tumor Registry. In some cases patients elected to receive follow up care with an ophthalmologist outside of the Duke Eye Center and for these patients limited data is available on their treatment outcomes. Therefore, the rates of local control, distant metastasis free survival and overall survival might be considered optimistic estimates since some patients may have experienced an event that was not recorded in our records. However, the length of follow up is actually most robust for those patients treated in the lowest dose quartile. Therefore any possible bias related to lost follow up would favor the higher dose quartiles with shorter follow up. This further supports our conclusions that those patients in the lower dose groups are unlikely to have worse outcomes.

In summary, in this retrospective analysis of 190 patients with uveal melanoma treated with episcleral ${ }^{125}$ I brachytherapy with doses ranging from less than $65 \mathrm{~Gy}$ to greater than $85 \mathrm{~Gy}$ to the tumor apex, we did not observe changes in local control or distant metastasis according to the dose prescribed to the apex. However, we did observe a significant increase in clinically meaningful ocular complications for patients treated with the upper quartiles of our dose range. Based on the results reported here, as well as reports from other series utilizing lower prescription doses [6,7], we propose a prospective multi-institutional study with well-coordinated long term follow up to evaluate rates of tumor control and ocular toxicity with lower prescription dose to the tumor apex. The cost and complexity of a randomized non-inferiority study would be significant; however a single-arm nonrandomized study prescribing lower dose to the tumor apex to evaluate outcomes may be 
more feasible. In the absence of data from a prospective trial, the available retrospective data from our study and from other investigators support prescribing a lower dose (i.e. $70 \mathrm{~Gy}$ ) to the tumor apex to treat uveal melanomas with ${ }^{125}$ I brachytherapy, particularly when the contralateral eye has poor visual function.

\section{Supplementary Material}

Refer to Web version on PubMed Central for supplementary material.

\section{Acknowledgments}

We would like to recognize critical contributions from medical physicists, Charles E. Nelson Ph.D. and Gerard M. Honore M.D. Ph.D., who helped to establish the episcleral plaque radiotherapy program at Duke in early 1980s. Without their efforts, this work would not have been possible. DGK was supported by K02 AI093866.

Dr. Kirsch reports grants, personal fees and stock/stock options from Lumicell, a company commercializing intraoperative imaging technology for cancer, which is outside the submitted work.

\section{References}

1. Singh AD, Turell ME, Topham AK. Uveal melanoma: Trends in incidence, treatment, and survival. Ophthalmology. 2011; 118:1881-1885. [PubMed: 21704381]

2. Stallard HB. Radiotherapy for malignant melanoma of the choroid. The British journal of ophthalmology. 1966; 50:147-155. [PubMed: 5909839]

3. Collaborative Ocular Melanoma Study G. The coms randomized trial of iodine 125 brachytherapy for choroidal melanoma: V. Twelve-year mortality rates and prognostic factors: Coms report no. 28. Archives of ophthalmology. 2006; 124:1684-1693. [PubMed: 17159027]

4. Melia BM, Abramson DH, Albert DM, Boldt HC, Earle JD, Hanson WF, Montague P, Moy CS, Schachat AP, Simpson ER, Straatsma BR, Vine AK, Weingeist TA. Collaborative Ocular Melanoma Study G. Collaborative ocular melanoma study (coms) randomized trial of i-125 brachytherapy for medium choroidal melanoma. I. Visual acuity after 3 years coms report no. 16. Ophthalmology. 2001; 108:348-366. [PubMed: 11158813]

5. Gragoudas ES, Lane AM, Regan S, Li W, Judge HE, Munzenrider JE, Seddon JM, Egan KM. A randomized controlled trial of varying radiation doses in the treatment of choroidal melanoma. Archives of ophthalmology. 2000; 118:773-778. [PubMed: 10865313]

6. Saconn PA, Gee CJ, Greven CM, McCoy TP, Ekstrand KE, Greven KM. Alternative dose for choroidal melanoma treated with an iodine-125 radioactive plaque: A single-institution retrospective study. International journal of radiation oncology, biology, physics. 2010; 78:844848.

7. Murray TG, Markoe AM, Gold AS, Ehlies F, Bermudez E, Wildner A, Latiff A. Long-term followup comparing two treatment dosing strategies of (125) i plaque radiotherapy in the management of small/medium posterior uveal melanoma. Journal of ophthalmology. 2013; 2013:517032. [PubMed: 23533708]

8. Design and methods of a clinical trial for a rare condition: The collaborative ocular melanoma study. Coms report no. 3. Controlled clinical trials. 1993; 14:362-391. [PubMed: 8222668]

9. Nath R, Anderson LL, Luxton G, Weaver KA, Williamson JF, Meigooni AS. Dosimetry of interstitial brachytherapy sources: Recommendations of the aapm radiation therapy committee task group no. 43. American association of physicists in medicine. Medical physics. 1995; 22:209-234. [PubMed: 7565352]

10. Nag S, Quivey JM, Earle JD, Followill D, Fontanesi J, Finger PT, American Brachytherapy S. The american brachytherapy society recommendations for brachytherapy of uveal melanomas. International journal of radiation oncology, biology, physics. 2003; 56:544-555.

11. Lee ET, Wang JW. Statistical methods for survival data analysis. ed. 
12. Cox DR. Regression models and life-tables. Journal of the Royal Statistical Society Series B (Methodological). 1972:187-220.

13. Diener-West M, Earle JD, Fine SL, Hawkins BS, Moy CS, Reynolds SM, Schachat AP, Straatsma BR. Collaborative Ocular Melanoma Study G. The coms randomized trial of iodine 125 brachytherapy for choroidal melanoma, ii: Characteristics of patients enrolled and not enrolled. Coms report no. 17. Archives of ophthalmology. 2001; 119:951-965. [PubMed: 11448318]

14. Kaliki S, Shields CL, Rojanaporn D, Badal J, Devisetty L, Emrich J, Komarnicky L, Shields JA. Scleral necrosis after plaque radiotherapy of uveal melanoma: A case-control study. Ophthalmology. 2013; 120:1004-1011. [PubMed: 23347983]

15. Jones R, Gore E, Mieler W, Murray K, Gillin M, Albano K, Erickson B. Posttreatment visual acuity in patients treated with episcleral plaque therapy for choroidal melanomas: Dose and dose rate effects. International journal of radiation oncology, biology, physics. 2002; 52:989-995.

16. Khan N, Khan MK, Bena J, Macklis R, Singh AD. Plaque brachytherapy for uveal melanoma: A vision prognostication model. International journal of radiation oncology, biology, physics. 2012; 84:e285-e290.

17. Almony A, Breit S, Zhao H, Garcia-Ramirez J, Mansur DB, Harbour JW. Tilting of radioactive plaques after initial accurate placement for treatment of uveal melanoma. Archives of ophthalmology. 2008; 126:65-70. [PubMed: 18195220] 


\section{Summary}

This retrospective study evaluates 190 patients with uveal melanoma prescribed I-125 plaque radiotherapy at varying doses (63-85 Gy). Increasing apex and $5 \mathrm{~mm}$ dose were correlated with greater visual acuity loss and radiation complications with no effect on tumor control outcomes. The Collaborative Ocular Melanoma Study Group (COMS) previously established $85 \mathrm{~Gy}$ to the tumor apex or $5 \mathrm{~mm}$ whichever is greater as standard prescribing practice. This study supports a prospective trial evaluating lower prescription doses. 


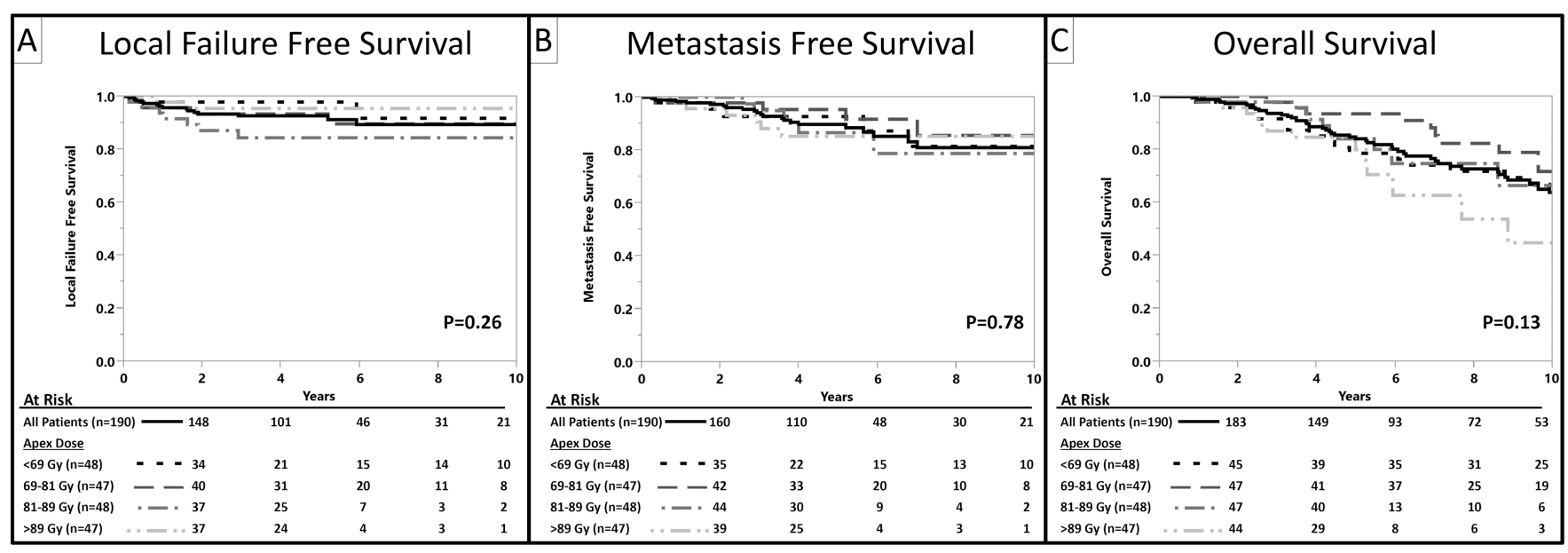

Figure 1. Treatment Outcomes with I-125 Plaques Among 190 Patients with Uveal Melanoma (A) Local Failure Free Survival by Apex Dose Quartile (B) Metastasis Free Survival by Apex Dose Quartile (C) Overall Survival by Apex Dose Quartile 


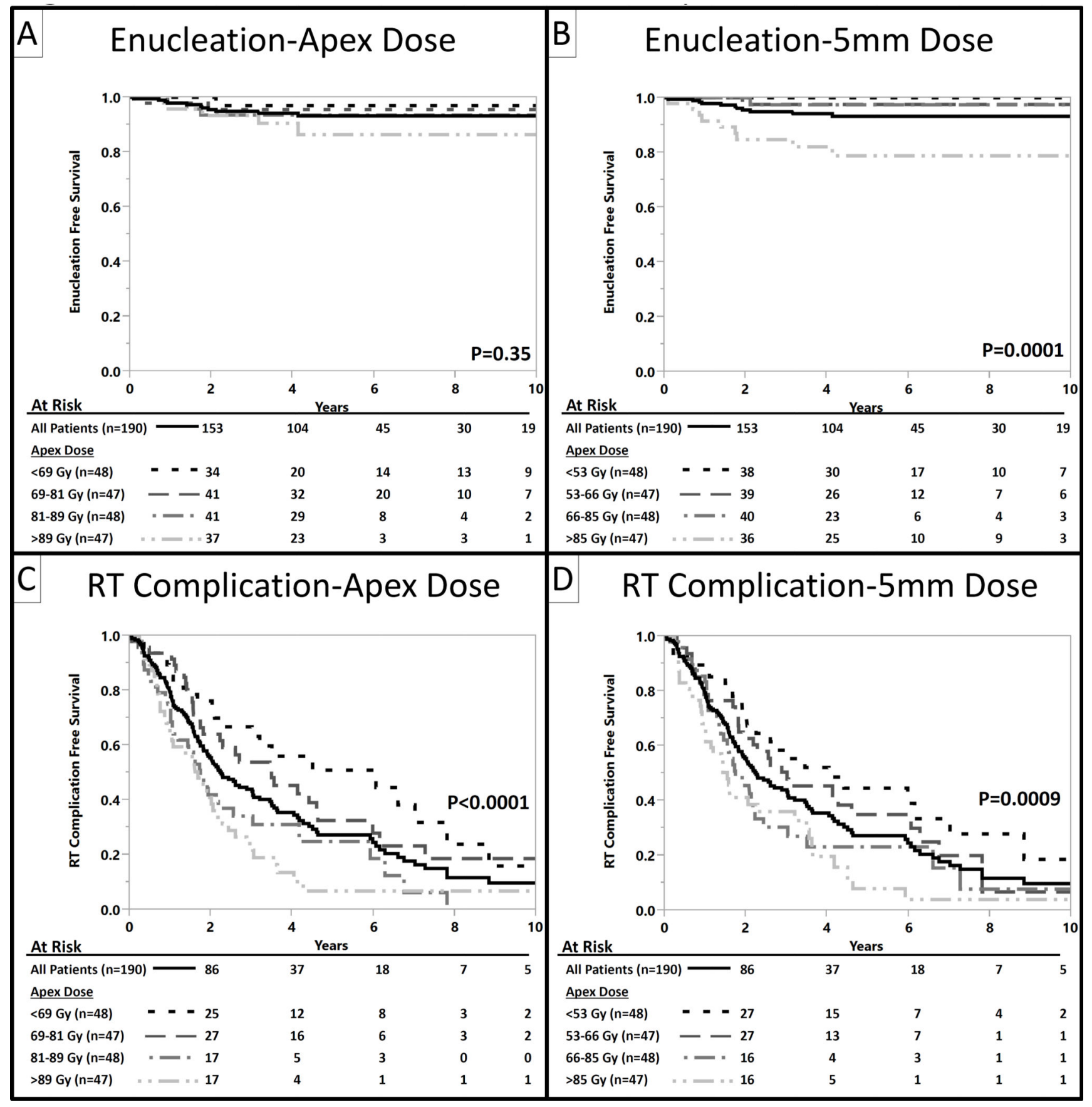

Figure 2. Eye Preservation and Radiation Treatment (RT) Complications with I-125 Plaques Among 190 Patients with Uveal Melanoma

(A) Enucleation Free Survival by Apex Dose Quartile (B) Enucleation Free Survival by 5 mm Dose Quartile (C) Any Radiation Related Toxicity Free Survival including retinopathy, optic neuropathy, cataract, neovascular glaucoma, and keratopathy by Apex Dose Quartile (D) Any Radiation Related Toxicity Free Survival by $5 \mathrm{~mm}$ Dose Quartile 


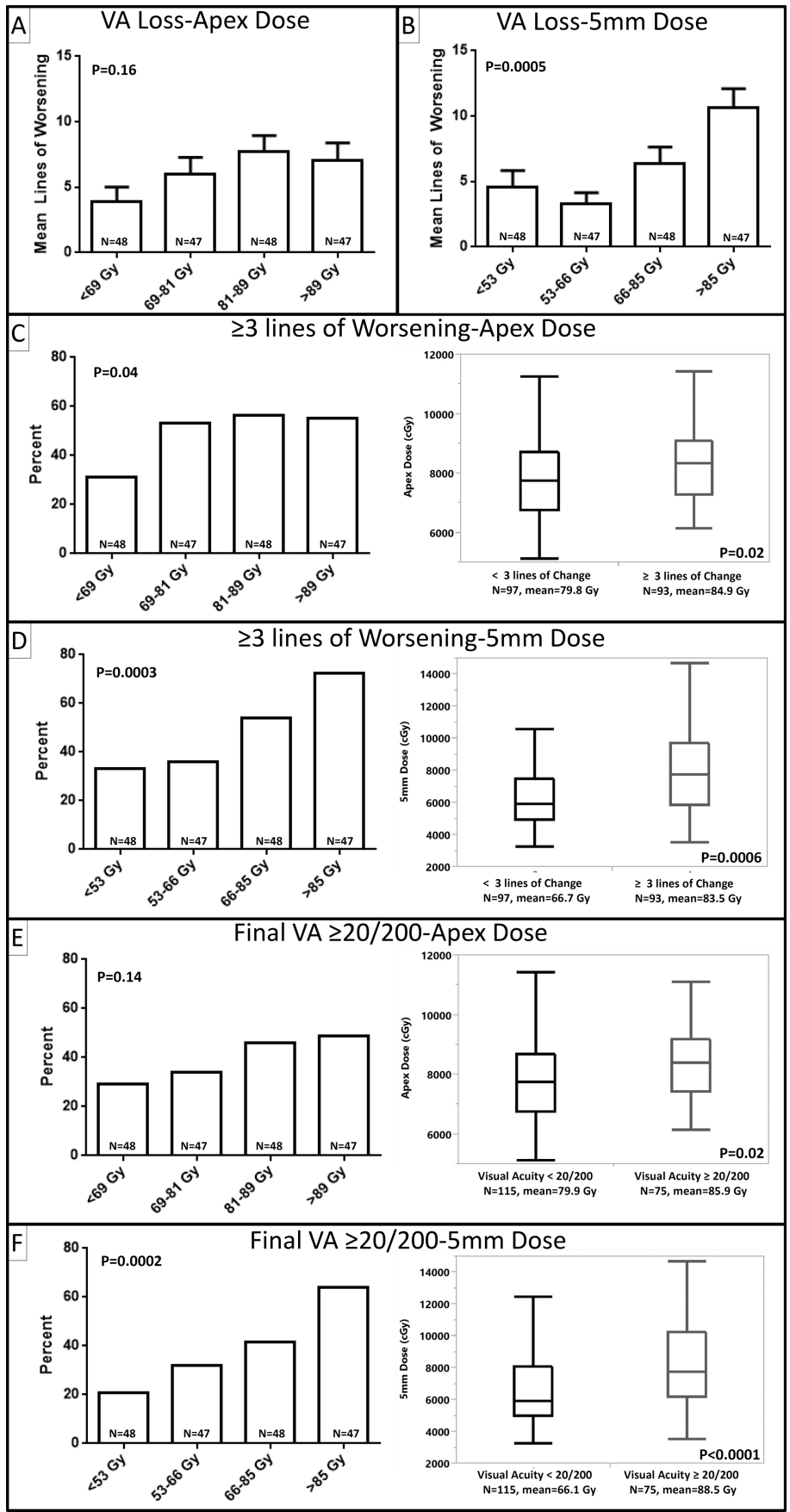

Figure 3. Visual Acuity with I-125 Plaques Among 190 Patients with Uveal Melanoma (A) Visual Acuity Loss by Apex Dose Quartile (B) Visual Acuity Loss by $5 \mathrm{~mm}$ Dose Quartile (C) Risk of greater than or equal to 3 lines of visual acuity loss by apex dose quartile (left) and apex dose among patients with less than 3 lines of visual acuity loss compared to greater than 3 lines of visual acuity loss (right) (D) Risk of greater than or equal to 3 lines of visual acuity loss by $5 \mathrm{~mm}$ dose quartile (left) and $5 \mathrm{~mm}$ dose among patients with less than 3 lines of visual acuity loss compared to greater than 3 lines of visual acuity loss (right) (E) Risk of final visual acuity worse than or equal to 20/200 by apex dose 
quartile (left) and apex dose among patients with visual acuity better than 20/200 compared to worse than or equal to 20/200 (right) (F) Risk of final visual acuity worse than or equal to 20/200 by $5 \mathrm{~mm}$ dose quartile (right) and $5 \mathrm{~mm}$ dose among patients with visual acuity better than 20/200 compared to worse than or equal to 20/200 (right) 
Table 1

Baseline Characteristics of Patients Treated with I-125 Brachytherapy between 1988-2010 at Duke University

\begin{tabular}{|c|c|}
\hline Demographic characteristics & $\begin{array}{r}\text { Number } \\
\text { (Percentage): }\end{array}$ \\
\hline Gender $(n=190)$ & \\
\hline Male & $92(48)$ \\
\hline Female & $98(52)$ \\
\hline Age $(\mathrm{yrs})(\mathrm{n}=190)$ & \\
\hline$<50$ & $42(22)$ \\
\hline $50-69$ & $96(51)$ \\
\hline 270 & $52(27)$ \\
\hline \multicolumn{2}{|l|}{$\underline{\text { Tumor/Treatment Characteristics }}$} \\
\hline Tumor apical height $(\mathrm{mm})(\mathrm{n}=190)$ & \\
\hline$<5$ & $130(69)$ \\
\hline $5-7.5$ & $48(25)$ \\
\hline $7.6-10$ & $10(5)$ \\
\hline$>10$ & $2(1)$ \\
\hline \multicolumn{2}{|l|}{ Longest basal dimension $(\mathrm{mm})(\mathrm{n}=190)$} \\
\hline$<8$ & $16(9)$ \\
\hline $8-11$ & $73(38)$ \\
\hline $11-14$ & $70(37)$ \\
\hline$>14$ & $31(16)$ \\
\hline Tumor Location $(\mathrm{n}=184)$ & \\
\hline ciliary body & $15(8)$ \\
\hline equator to ora serrata & $39(21)$ \\
\hline posterior to equator not involving macula & $52(28)$ \\
\hline posterior to equator involving macula & $78(43)$ \\
\hline \multicolumn{2}{|l|}{ Tumor Quadrant ( $\mathrm{n}=181)$} \\
\hline superotemporal & $54(30)$ \\
\hline inferotemporal & $65(36)$ \\
\hline superonasal & $29(16)$ \\
\hline inferonasal & $33(18)$ \\
\hline \multicolumn{2}{|l|}{ Plaque Type $(\mathrm{n}=183)$} \\
\hline circular & $120(66)$ \\
\hline notched & $43(23)$ \\
\hline ciliary & $20(11)$ \\
\hline
\end{tabular}




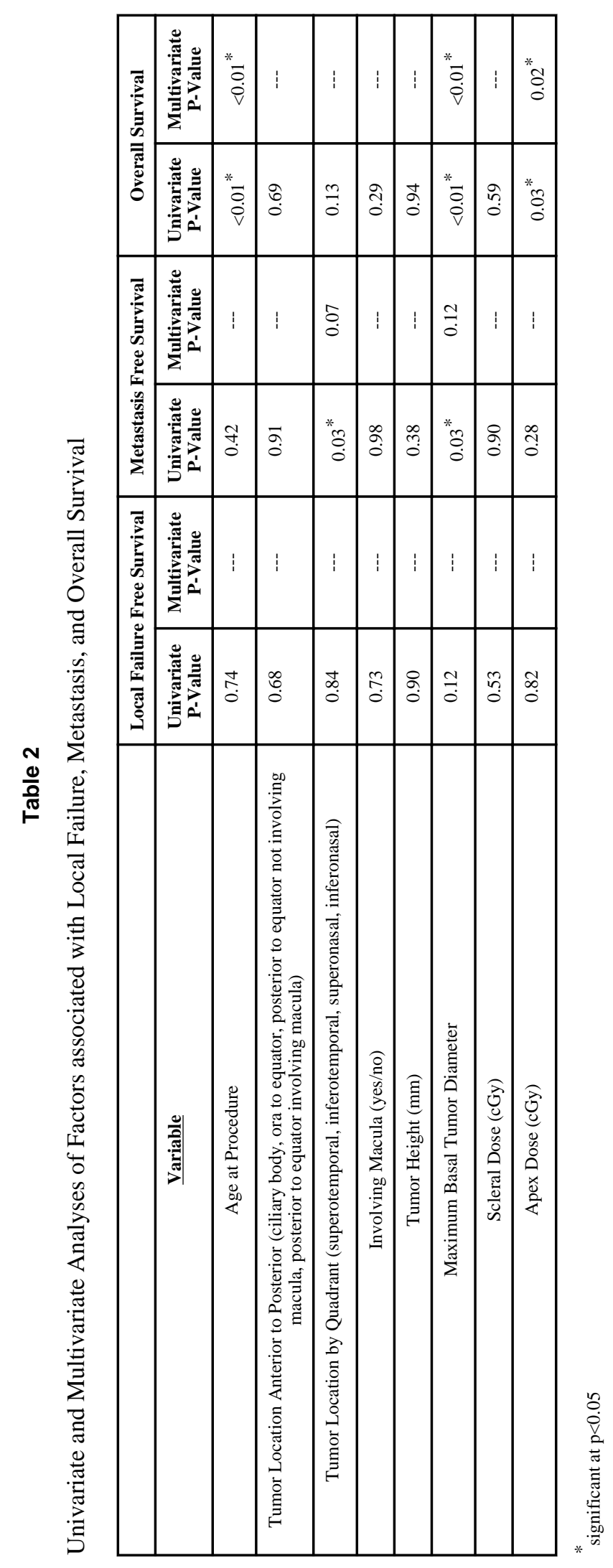


Table 3

Univariate and Apex Dose Multivariate Analysis of Factors associated with Radiation Toxicity and Enucleation

\begin{tabular}{|c|c|c|c|c|}
\hline Variable & Any Radiation Toxicity & \multicolumn{2}{|c|}{ Enucleation } \\
\hline Age at Procedure & $\begin{array}{c}\text { Univariate } \\
\text { P-Value }\end{array}$ & $\begin{array}{c}\text { Multivariate } \\
\text { P-Value }\end{array}$ & $\begin{array}{c}\text { Univariate } \\
\text { P-Value }\end{array}$ & $\begin{array}{c}\text { Multivariate } \\
\text { P-Value }\end{array}$ \\
\hline $\begin{array}{c}\text { Tumor Location Anterior to Posterior (ciliary body, ora to equator, posterior to } \\
\text { equator not involving macula, posterior to equator involving macula) }\end{array}$ & 0.46 & --- & 0.66 & --- \\
\hline $\begin{array}{c}\text { Tumor Location by Quadrant (superotemporal, inferotemporal, superonasal, } \\
\text { inferonasal) }\end{array}$ & 0.17 & --- & $0.03^{*}$ & 0.09 \\
\hline Involving Macula & --- & 0.90 & -- \\
\hline Tumor Height (mm) & 0.14 & --- & 0.42 & $-\ldots$. \\
\hline Maximum Basal Tumor Diameter & $<0.01^{*}$ & $<0.01^{*}$ & $<0.01^{*}$ & $<0.01^{*}$ \\
\hline Apex Dose (cGy) & 0.37 & --- & 0.12 & --- \\
\hline
\end{tabular}

significant at $\mathrm{p}<0.05$ 